\title{
Physiotherapy Approach towards a Typical Case of Injection Induced Radial Nerve Palsy Presenting as Wrist Drop
}

\author{
Dushyant Padmakar Bawiskar¹, Anjali Sunil Bais², Waqar M. Naqvi³ ${ }^{3}$ Arti Sahu ${ }^{4}$ \\ ${ }^{1}$ Department of Physiotherapy, Datta Meghe Institute of Medical Sciences (Deemed to be University), \\ Sawangi, Meghe, Wardha, Maharashtra, India. ${ }^{2}$ Department of Physiotherapy, Datta Meghe Institute \\ of Medical Sciences (Deemed to be University), Sawangi, Meghe, Wardha, Maharashtra, India. \\ ${ }^{3}$ Department of Physiotherapy, Datta Meghe Institute of Medical Sciences (Deemed to be University), \\ Sawangi, Meghe, Wardha, Maharashtra, India. ${ }^{4}$ Department of Physiotherapy, Datta Meghe Institute \\ of Medical Sciences (Deemed to be University), Sawangi, Meghe, Wardha, Maharashtra, India.
}

\section{INTRODUCTION}

Wrist drop occurs as a consequence of radial nerve palsy. From the estimates, it is found that each person receives at least two injections annually in the developing countries, and 50 percent of these injections are not considered safe.(1) Unfortunately, trauma to peripheral nerves isn't unusual in our country. Though reliable statistics are not available at the national level, the incidence of injury to peripheral nerves, especially to radial nerve, is on the rise, largely due to increased road traffic accidents and increased violence in our society as a whole.(1) Radial Nerve damage occurring as a consequence of intramuscular injection application is comparatively less common than sciatic nerve injury.(2) When therapeutic and prophylactic agents are applied in the form of intramuscular injections into buttock and arm, occasionally damage to the peripheral nerves can occur. Those whose radial nerve function has been compromised would not be able to actively extend extensor muscles of the wrist and digits since the radial nerve innervates them. As a result, the hand hangs flaccidly in a flexed position when the patient attempts to move the arm to a horizontal position. Patients may also experience paraesthesia, hypoesthesia, numbness and other sensory disturbances. Simple, pain-free sensory deficits may be functionally less significant because they involve the anatomical snuffbox and the radial dorsum of the hand. Injury to radial nerve results in motor function impairment leading to weakness during extension of the forearm, wrist, and fingers. However, wrist extension may be relatively spared because common radial nerve innervates the extensor carpi radialis longus muscle proximal to its division.(3)

The inability of a person to extend his wrist and/or fingers due to the above mentioned causes is called "wrist drop" or "finger drop."(4) The other causes of wrist drop include stab wound injury to shoulder area where the terminal branch of posterior cord of brachial plexus is given off as radial nerve and any agent causing persistent injury to nerve. Interventions for wrist drop include splinting, electrical stimulation, exercises for hand and fingers, surgery, and drug therapy. The present case is diagnosed with Wrist Drop caused due to the injection induced injury to the radial nerve of left side. Needle length recommendation is very important before inducing the injection in male and female.(4)

In the developing countries, common diseases are treated with the application of injections. When therapeutic and prophylactic agents are applied in the form of intramuscular injections into buttock and arm, occasionally damage to the peripheral nerves can occur. There are various reasons for the injury to the peripheral nerves. In case of wrist drop, reasons for the injury to the radial nerve may be gun-shot injury, humeral shaft fracture, misplaced injection and compression or ischemia.
Corresponding Author: Dr. Waqar M. Naqvi, Professor and HOD, Community Health Physiotherapy, Ravi Nair Physiotherapy College, Wardha, Maharashtra, India.

E-mail:waqar.naqvi@dmimsu.edu.in

DOI: $10.14260 /$ jemds $/ 2020 / 378$

Financial or Other Competing Interests: None.

How to Cite This Article:

Bawiskar DP, Bais AS, Naqvi WM, et al. Physiotherapy approach towards a typical case of injection induced radial nerve palsy presenting as wrist drop. J. Evolution Med. Dent. Sci. 2020;9(22):1722-1724, DOI: 10.14260/jemds/2020/378

Submission 26-03-2020,

Peer Review 10-05-2020,

Acceptance 16-05-2020,

Published 01-06-2020.

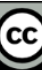




\section{PRESENTATION OF CASE}

A unique case of Radial Nerve Palsy was reported in a farmer resulting as a consequence of injection induced palsy. No surgical interventions were given to the patient. The injury was caused by tip of the needle of the injection. The symptoms included pain over the shoulder, which was immediate, sharp and electric-like. He also presented with numbness, paraesthesia, hypothesis and other sensory disturbance and weakness while extending the wrist and fingers. Treatment included proper skin care, stretching, orthotic device (static \& dynamic), and electrical stimulation for muscle re-education. It is very important for a Healthcare professional to take proper measures to avoid such nerve injury.

\section{Patient Information}

A 51-year-old male patient, farmer by occupation with body mass index of $26 \mathrm{~kg} / \mathrm{m}^{2}$ and right-hand dominant was referred into Physical therapy Department for rehabilitation. Patient was absolutely alright till 26/12/19, and then he experienced pain over left shoulder due to heavy lifting. Pain was sudden in onset, gradually progressive, continuous in nature, and not associated with tingling and numbness. With this complaint, he visited the private hospital where he was prescribed medications and an injection was induced in the region of deltoid muscle for the left shoulder pain. Immediately after the application of injection, he experienced sharp shooting pain at the injection site which radiated to the back of his forearm. He also experienced numbness, loss of sensation in the $1^{\text {st }}$ and $2^{\text {nd }}$ digits and loss motor function of the extensor compartment of forearm of left hand. With all this complaint, he was referred to the Physical Therapy Department. There was no relevant any past history, family history, Drug History.

\section{Clinical Findings}

On examination, muscle power of left side fingers extensors are $0 / 5$, left side wrist extensor are $0 / 5$, elbow flexors are $4 / 5$ and shoulder flexors and extensor are $4 / 5,4 / 5$ respectively according to medical research council.

\begin{tabular}{|c|c|c|}
\hline & Active ROM & Passive Rom \\
\hline Shoulder flexion & $0-180$ degree & $0-185$ degree \\
\hline Shoulder extension & $0-40$ degree & $0-4$ \\
\hline Elbow Flexion/Extension & -135 degree flexion/ 0 degree & $\begin{array}{c}0-140 \text { degree flexion/ } 0 \\
\text { degree }\end{array}$ \\
\hline $\begin{array}{l}\text { Forearm Pronation/ } \\
\text { Supination }\end{array}$ & $0-65$ degree & 0-67 degree/ 0-79 degree \\
\hline Wrist Extension/ flexion & 0 degre & $0-65 c$ \\
\hline Wrist Radial/ Ulnar & 0 degree / 0 -30 degree & 0-15 degree/ 0-35 degree \\
\hline \multicolumn{3}{|c|}{ Assessment of Range of Motion of the Affected Side } \\
\hline
\end{tabular}

On neurological examination, impaired sensation in the C6-C7 and C8 dermatomal region of Left Hand was reported.

His complete routine examination was normal. Patient underwent diagnostic test for radial nerve i.e. Nerve Conduction Velocity Test as an outcome measure. The reports showed a reduction in nerve conduction in left radial nerve.

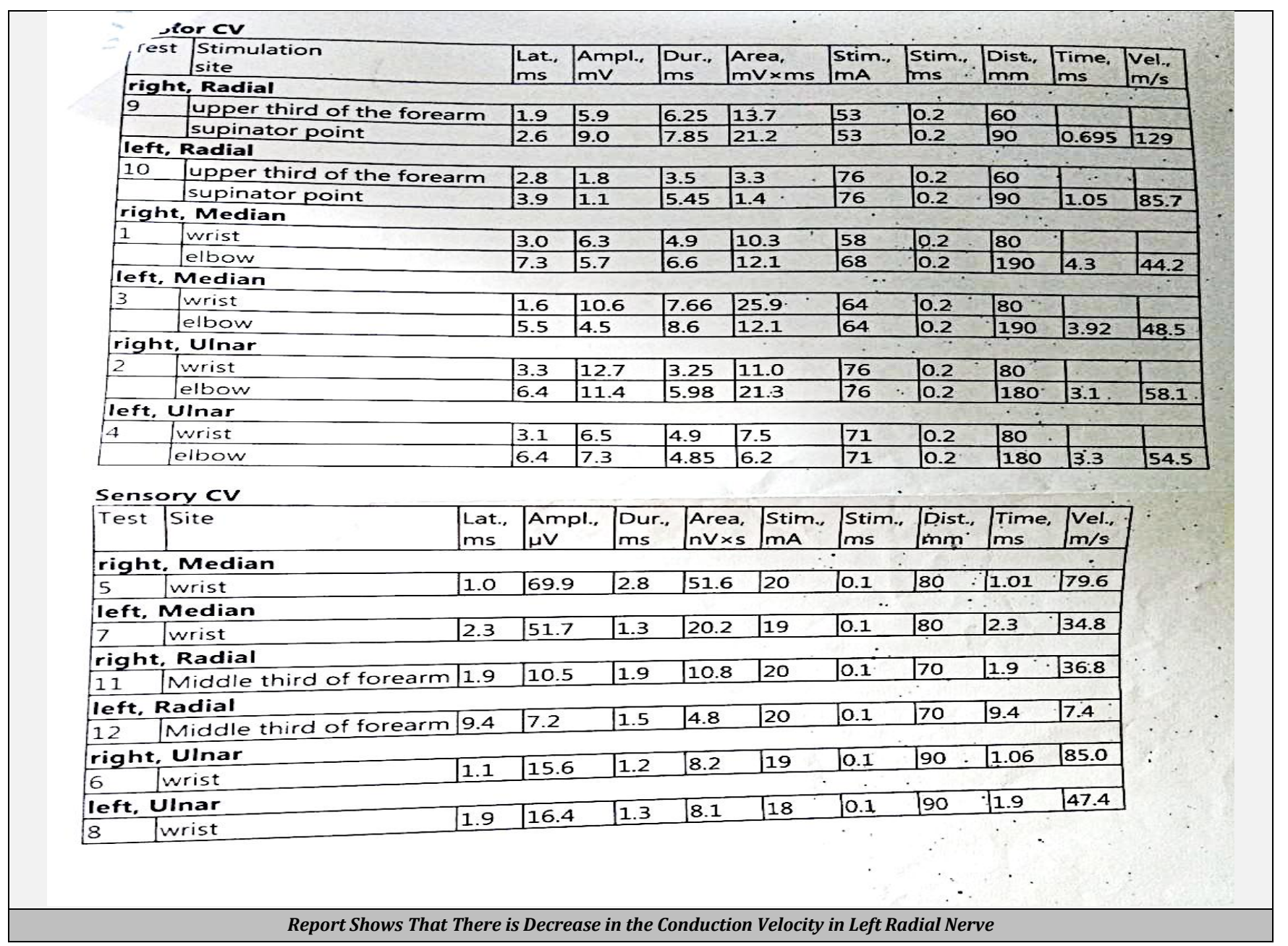




\section{DISCUSSION OF MANAGEMENT}

Therapeutic interventions were started from the first day of patient visit. Initially, cock-up splint was prescribed to maintain the wrist in extended position.(1) Full passive range of motion exercises were initiated for the wrist and hand joints. Patient was educated about the assisted active exercises for extension of wrist, fingers and thumb, elbow flexion in supination and forearm supination. Stretches were taught for finger joints, wrist joint and elbow joint of the left side and were performed in 2 sets where in 5 repetitions were performed with 15 seconds hold in between simultaneous repetitions. Proper care of the limb was advised to the patient in order to prevent the skin from infection along with proper inspection to look for any bruises. As the skin type is dry, patient was informed to apply moisturizing ointment over that region.

Electrical muscle stimulation was given to the affected muscles (current type-Intermittent Galvanic; WavelengthTriangular; duration-30-30-30 stimulation) to facilitate effective muscle education. Visual Feedback exercises were advised to the patient in order to improve the position of hand.

On the $45^{\text {th }}$ day of rehabilitation, patient reported slight voluntary movement of left side fingers and wrist along with an increase in the sensation as compared to day 1. Muscle power of left side fingers extensor was $1 / 5$, left side wrist extensor was $1 / 5$, elbow flexors was $4 / 5$ and shoulder flexors and extensor was $4 / 5$ and $4 / 5$ respectively according to medical research council.

\section{DISCUSSION}

Intramuscular injection can cause injury to a peripheral nerve resulting from the piercing of needle, or from the solution used for injection into the nerve. Potential injuries to the radial nerve are of significant concern due to high frequency of in administering intramuscular injections. While some patients recover spontaneously with the conservative treatment, others may require electrical muscle stimulation in addition to the conservative treatment for better prognosis.

Since patient was highly motivated, he performed the exercises with enthusiasm. He was proactive in performing the exercises prescribed at the Rehabilitation Centre. He also carried out the home exercise programme sincerely. The limitation to the rehabilitation included long duration of treatment and patient's inability to pay the medical expenses for the treatment. Appropriate duration and frequency of stretching plays a significant role because stretching with duration of 30 seconds has been found most effective.

NCV test was done for the radial nerve as an outcome measure. CMAP amplitude was reduced with normal distal motor latency and conduction velocity in Left Radial Nerve and SNAP amplitude was reduced with normal conduction velocity in left radial nerve. Post treatment NCV was not performed due to financial limitation of the patient but treatment continued for the management of the radial nerve palsy.

\section{REFERENCES}

[1] Ljungquist KL, Martineau P, Allan C. Radial Nerve Injuries. J Hand Surg 2015;40(1):166-72.

[2] Pandian JD, Bose S, Daniel V, et al. Nerve injuries following intramuscular injections: a clinical and neurophysiological study from Northwest India. J Peripher Nerv Syst 2006;11(2):165-71.

[3] Hobson-Webb LD, Juel VC. Common Entrapment Neuropathies. Continuum (Minneap Minn) 2017;23(2, Selected topics in Outpatient Neurology):487-511.

[4] Kim HJ, Park SK, Park SH. Upper limb nerve injuries caused by intramuscular injection or routine venipuncture. Anesth Pain Med 2017;12(2):103-10. 